\title{
AN IMPROVED TYPE OF WAVE METER RESONANCE INDICATOR
}

\author{
By Morris S. Strock
}

ABSTRACT

A resonance indicator as used on most portable wave meters commonly consists of a "thermogalvanometer" connected in series in the tuncd circuit of the wave meter. The use of this instrument reduces the precision of setting the wave-meter condenser, because it adkls to the resistance of the wave meter and because it gives comparatively small resonance deflections at low settings of the condenser.

This paper describes a resonance indicator for a portable wave meter which can be used in place of the thermogalvanometer. It gives an approximately uniform resonance deflection over the frequency range of the wave meter, does not appreciably increase its resistance, and is more sensitive than the thermogalvanometer. The method employs a sensitive milliammeter and a crystal detector connected in such a manner that a combination of capacitive and inductive coupling is obtained with respect to the wave-meter circuit.

The operation of a wave meter: equipped with this device is not quite as simple as the operation of a wave meter equipped with a thermogalvanometer, because it requires an adjustment of the crystal detector. Horwever, if the detector is of good mechanical design and is provided with a crystal of uniform sensitivity this is not a great drawback. Experimental data are given to show that this method of resonance indication permits precise settings of the wave-meter condenser. The resonance indicator may be added to most portable wave meters and results in greater precision in their use.

\section{CONTENTS}

I. Introduction

Page

II. Principle of operation

111

III. Method of application

IV. Results

V. Summary and conclusions

117

\section{INTRODUCTION}

Most portable wave meters contain a visual resonance indicator to show when the wave-meter circuit is tuned to resonance with the source of radio-frequency power. This indicator commonly consists of a "thermogalvanometer" connected in series in the tuned circuit $20672^{\circ}-25$ 
of the wave meter. The use of such an instrument as a resonance indicator has the following disadvantages:

1. The thermogalvanometer has a resistance of several ohms which increases the radio-frequency resistance of the wave meter, resulting in broader tuning, which reduces the precision of setting the wavemeter condenser.

2. The resonance deflections of the thermogalvanometer are comparatively small at low settings of the wave-meter condenser, and if the frequency of a source of low power is being measured these small deflections again affect the precision of setting the wave-meter condenser.

The first objection could be eliminated by removing the thermogalvanometer from the wave-meter circuit and connecting its terminals to a pick-up coil coupled to the wave-meter coil. This method

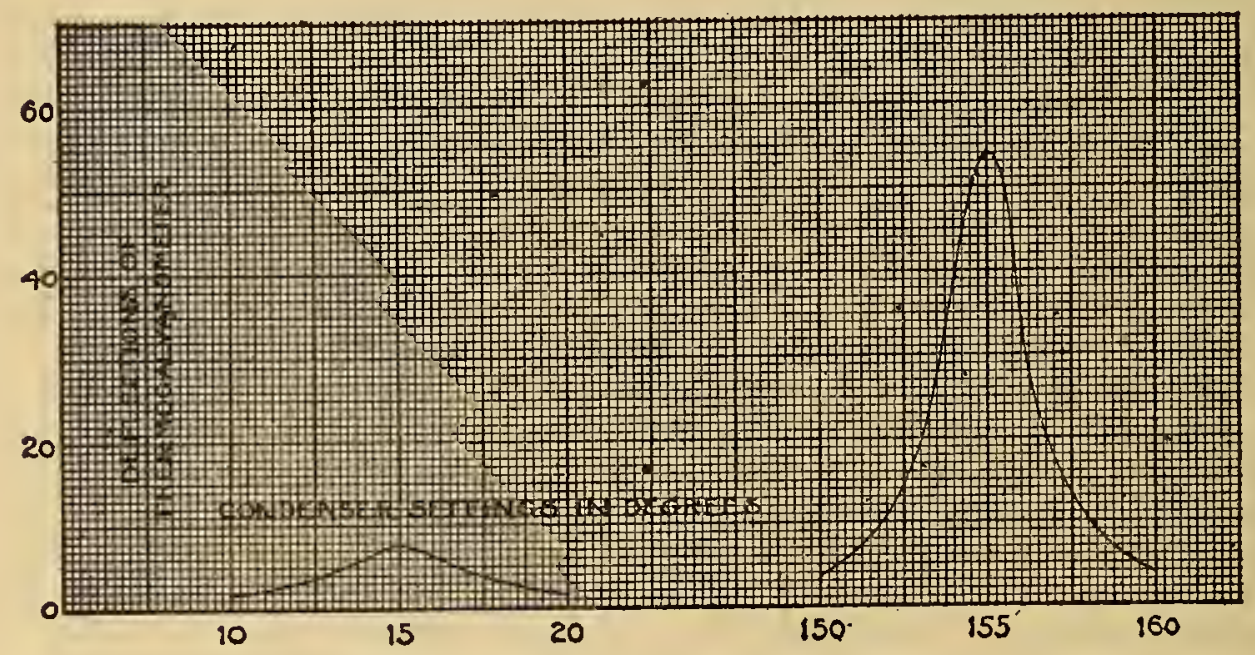

FIG. 1.-I'ypical resomance curves of wave meter equipped with thermogalvanometer

is, however, unsuitable for use on a portable wave meter with exposed coils because it is necessary to provide a mounting and connection terminals for the pick-up coil so that it can be removed when the wave meter is closed for transportation. Furthermore, this method does not overcome the second objection.

The second objection merits further consideration because at first sight it might appear that it would make no difference whether the indicator showed large or small resonance deflections as long as these deflections could be readily noted. It can be shown, however, that a greater deflection means greater precision in setting the wave-meter condenser. In Figure 1 the two resonance curves, plotted with thermogalvanometer deflections as ordinates and condenser settings as abscissas, were obtained at two different points on the condenser scale using constant coupling and constant radiated power in the 
generator. The curves show the relatively small deflections obtained at low settings of the wave-meter condenser, and the higher curve having a greater slope near its peak shows that for a given change of the condenser a greater change in deflection, hence greater precision, is obtained. The small deflections obtained at the lower condenser settings can be increased by using closer coupling, which however, can not be increased beyond a certain amount because the reactive effect between the wave meter and the generator impairs the accuracy of the measurement.

A resonance indicator has been developed which does not have the disadvantages of the thermogalvanometer and which is suitable for use on a portable wave meter. This indicator employs a crystal detector and a sensitive direct-current milliammeter coupled to the wave-meter circuit in a special manner. The use of a detector adds another adjustment to the wave meter, but if a properly designed detector is used this disadvantage is not a great drawback. The advantages of this device are: (1) It does not appreciably increase the radio-frequency resistance of the wave-meter circuit. (2) It gives approximately the same resonance deflections over the frequency range of the wave meter.

It is the purpose of this paper to describe the principle of operation, the method of application, and the results obtained by this method of indicating resonance.

\section{PRINCIPLE OF OPERA- TION}

In Figure 2 is shown a wavemeter circuit consisting of coil $L$
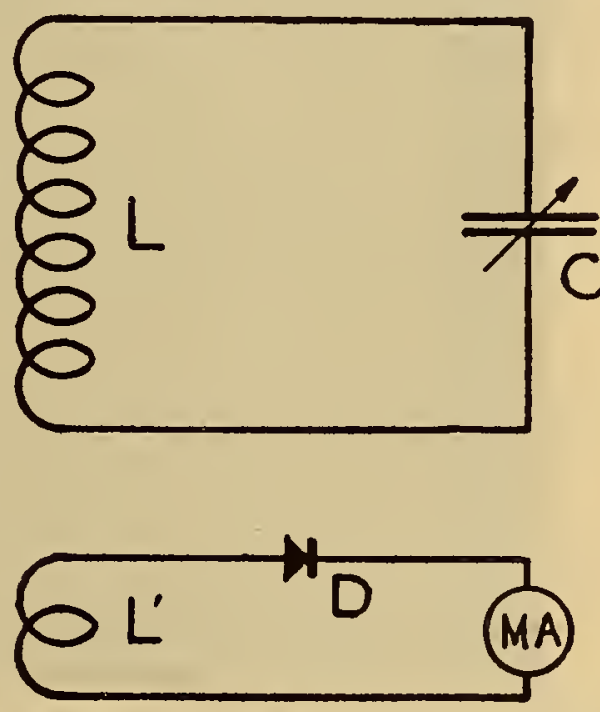

Fig. 2.-Connections of simple crystal milliammeter resonance indicator.

and condenser $C$. A pick-up circuit (untuned), which consists of a coil $L^{\prime}$, a crystal detector $D$, and a milliammeter $M A$, having a full-scale deflection of 1 or 2 milliamperes, is coupled to the wavemeter circuit. When the wave-meter circuit is tuned to resonance with the radio-frequency power source a maximum current is set up which induces an alternating emf in $L^{\prime}$ and the resultant current is rectified by the crystal $D$, so that a deflection is obtained on the milliammeter. In this simple form the resonance indicator is not suitable for use on a portable wave meter because external coupling is required. It is also true that this method does not give uniform resonance deflections over the frequency range of the wave meter. 
The system developed for a portable wave meter employs the principle just noted and, in addition, a combination of capacitive and inductive coupling, resulting in advantages (1) and (2) given above. The following description shows how this development was made:

The pick-up circuit of Figure 2 was experimentally applied to a portable wave meter using a coil of a few turns for $L^{\prime}$ and a milliammeter $M A$ having full-scale deflection of 1 milliampere. A number of settings were chosen for the wave-meter condenser, and the generator was successively tuned to resonance with the several condenser settings. Resonance deflections of milliammeter $M A$ were then recorded for various settings of condenser $C$, and a curve

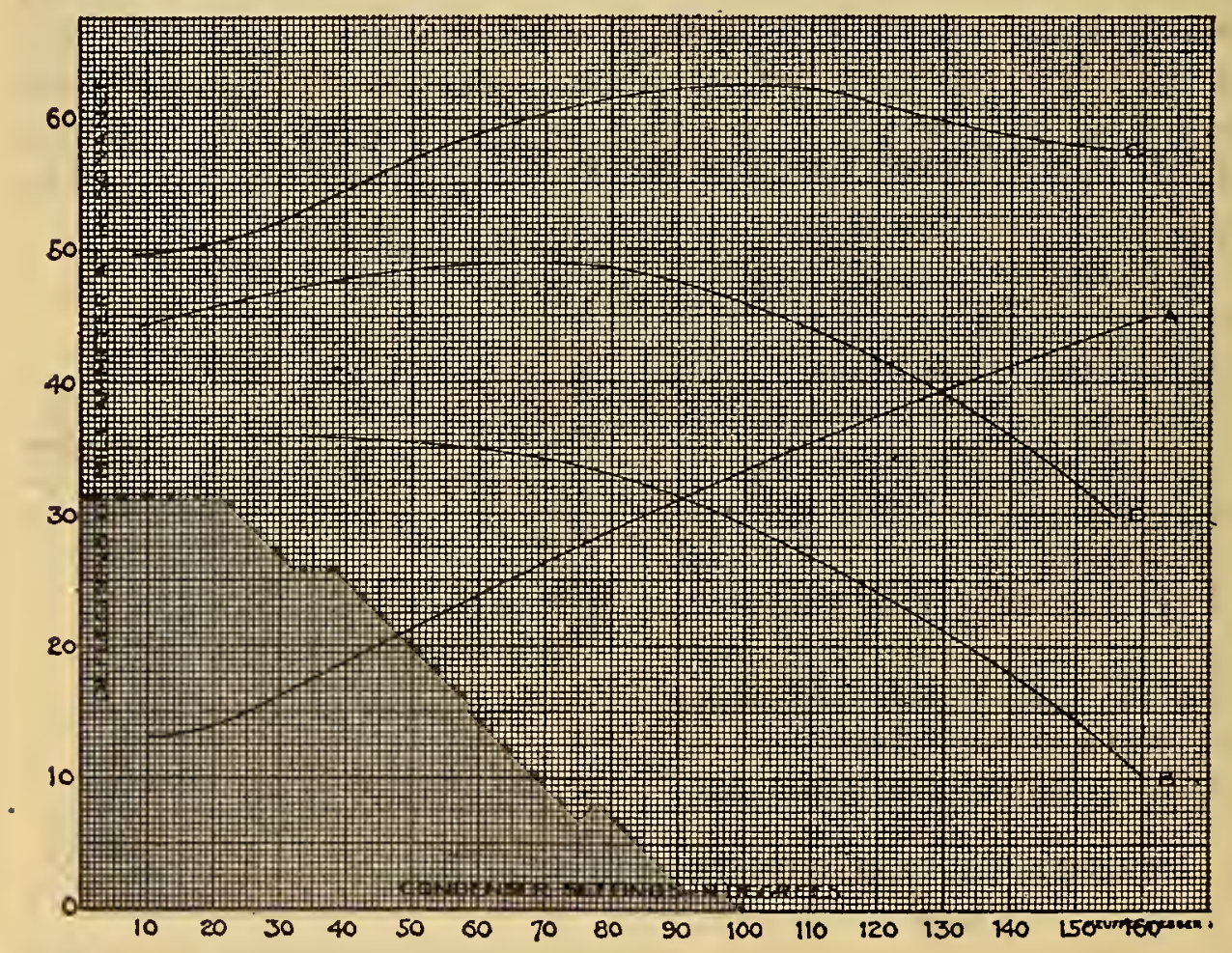

FrG. 3.-Curves illustrating variations in resonance deflctions obtained from circuits employing differcnt connections for crystal and milliammeter

$A$ (fig. 3) was obtained. In obtaining this curve the generator was adjusted so that the radiated power remained constant. This curve was obtained at frequencies between 500 and $1,000 \mathrm{kc}$. If plotted over a different band of frequencies, its slope varies considerably.

A curve somewhat similar to $A$ (fig. 3) would be obtained for a wave meter equipped with a thermogalvanometer. Such a curve would be closer to the axis of abscissas because smaller deflections are obtained at resonance. Increased deflections for curve $A$ may be obtained by using more turns on $L^{\prime}$ (fig. 2), but too many turns absorb so much power from the wave-meter circuit that a readjust- 
ment of the crystal detector changes the wave meter calibration. ${ }^{1}$ Greater sensitivity can be obtained by substituting a large type of millivoltmeter for $M A$, but this instrument is unsuitable for a portable wave meter.

Connections were next made as indicated in Figure 4. The wire leading from the condenser terminal to the detector is usually called a one-point connection, and it was taken from the fixed plates of the wave-meter condenser. This connection supplies capacitive coupling. A curve plotted from this system gave curve $B$ (fig. 3).

Curve $A$ shows that the increasing resonant deflections of the milliammeter are obtained at increasing condenser settings, and since the wave-meter circuit has a decreasing resistance with increasing condenser settings curve $A$ may be said to represent the current

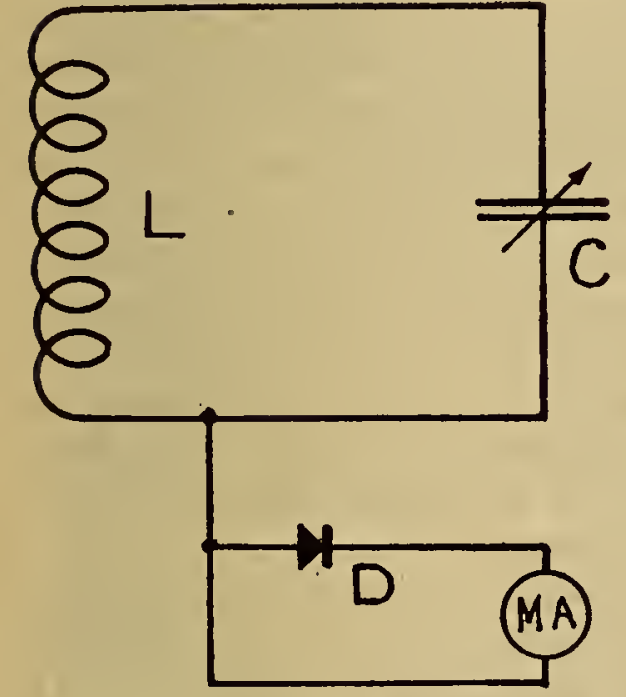

FTa.4.-One-point connection of resonance indicator

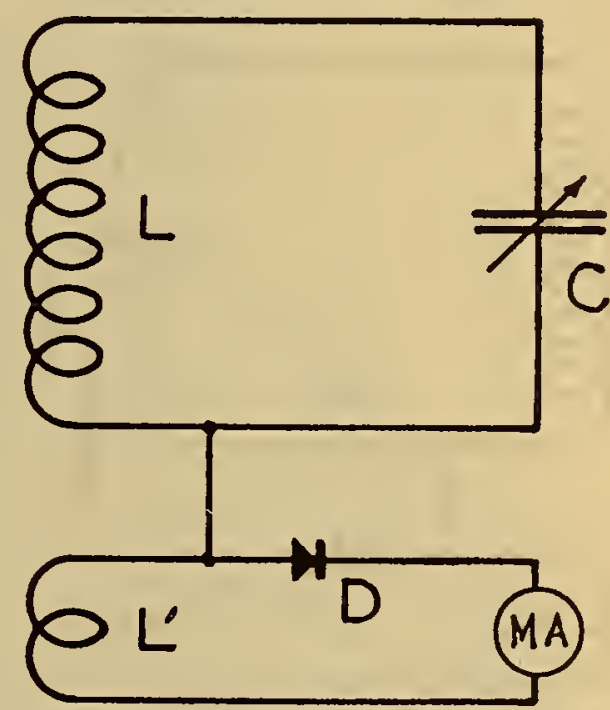

FiG. 5.-Connections for resonance indicator which give approximately uniform resonance deflections.

effect of the wave meter. On the other hand, curve $B$ shows that increasing resonant deflections of the milliammeter are obtained with decreasing condenser settings, and since the voltage across the wave-meter condenser increases as the condenser setting decreases curve $B$ may be said to represent the voltage effect of the wave meter.

When the ordinates of curves $A$ and $B$ (fig. 3) are added, a resultant curve $C$ is obtained which represents approximately the same resonance deflections of the milliammeter at all condenser settings. Thus, curve $C$ is the theoretical curve which might result by combining the circuit of Figure 2 with that of Figure 4, as shown in

1 Here the use of a fixed cryștal detector suggests itself, but this device is unsuitable because its adjustment changes with time. 
Figure 5. This circuit uses a combination of capacitive and inductive coupling. Actual observed data from this new circuit gave the curve $D$ (fig. 3). By making many tests the circuit $L^{\prime} D M A$. (fig. 5) could, no doubt, be so designed that curve $D$ (fig. 3) would be very nearly a straight line parallel to the axis of abscissas. This ideal result is, however, unnecessary; in fact, it is an advantage to have the curve rise slightly with decreasing condenser settings.

\section{METHOD OF APPLICATION}

In applying the circuit of Figure 5 to a portable wave meter $L^{\prime}$ must be placed in the wave-meter case. A coil of a few turns is not suitable for this purpose, but experiments showed that a small coil of many turns placed in the field of the condenser gave the required

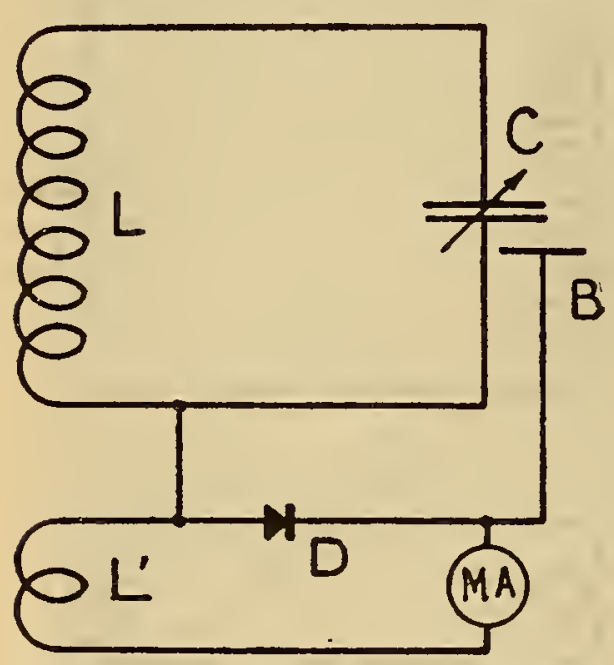

Fig. 6.-Connections for resonance indicator as finally adopted. amount of inductive coupling. The proper size of this coil is determined experimentally. To assist in securing approximately uniform resonance deflections over the frequency range of the wave meter, it is desirable to supply additional capacitive coupling by means of a metal plate connected as shown at $B$, Figure 6. The size and position of $B$ are also determined experimentally.

Figures $7 \mathrm{a}$ and $7 \mathrm{~b}$ show a portable wave meter changed to employ the connections of Figure 6. $L^{\prime}$ (fig. 6) consists of about 20 turns of wire wound on an insulating material form as shown at $A$, Figure 7a. $B$ is a copper sheet held in place by the form $A$. The milliammeter is attached to binding posts $C$ of the wave meter, which were originally used for a telephone receiver. The milliammeter is of a small portable type giving a fullscale deflection of 1 milliampere. A damping resistance is connected across the terminals of the milliammeter. This reduces the deflections, but it prrents excessive swinging of the needle.

\section{RESULTS}

The wave meter shown in Figures $7 \mathrm{a}$ and $7 \mathrm{~b}$ is in use at this bureau and has proved to be very satisfactory. The results of special tests, indicating the precision which may be obtained from frequency measurements with this wave meter, are given in the table 
Scientific Papers of the Bureau of Standards, Vol. 20

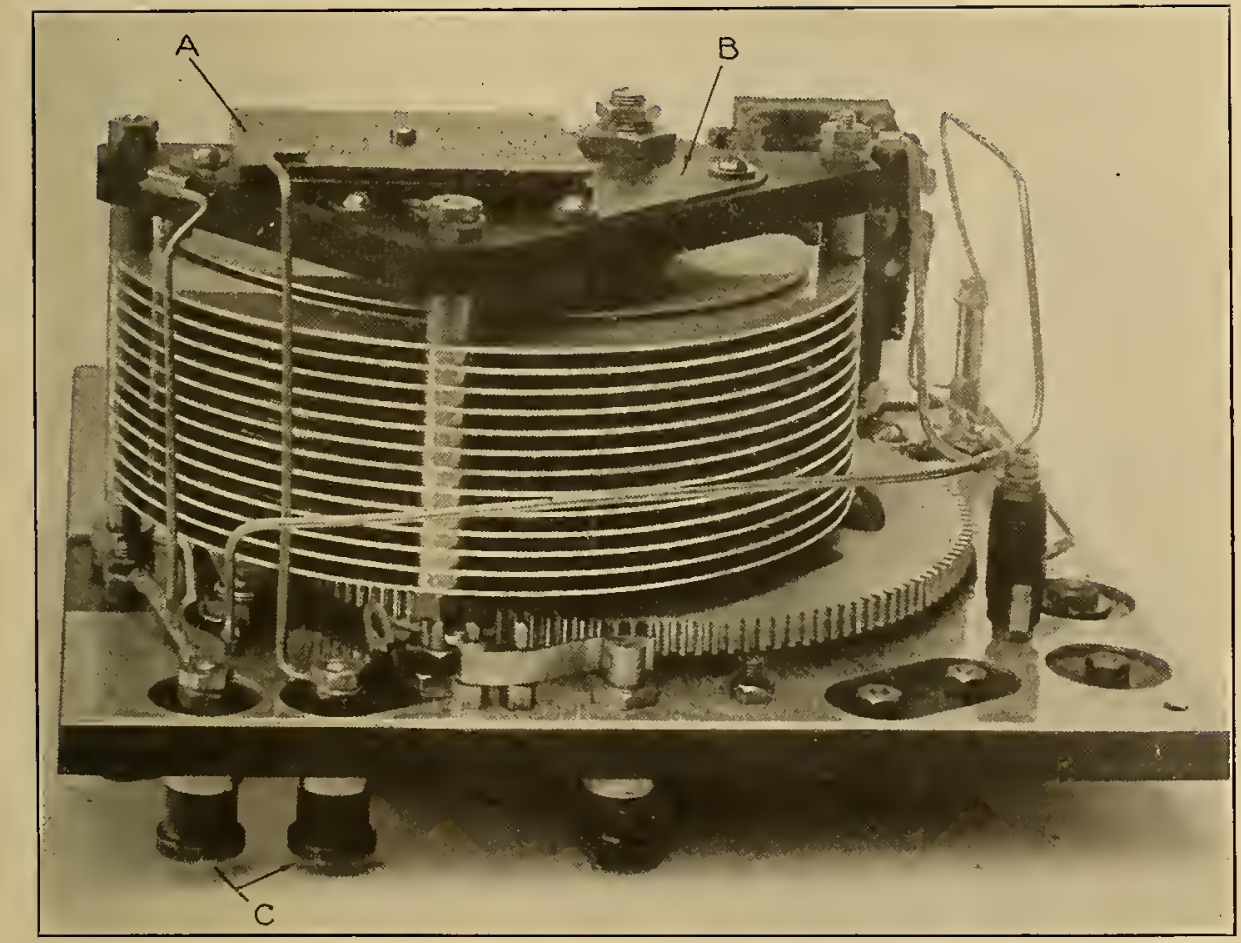

FIG. 7a.-Interior view of wave meter equipped with crystal milliammeter resonance indicator 
Scientific Papers of the Bureau of Standards, Vol. 20

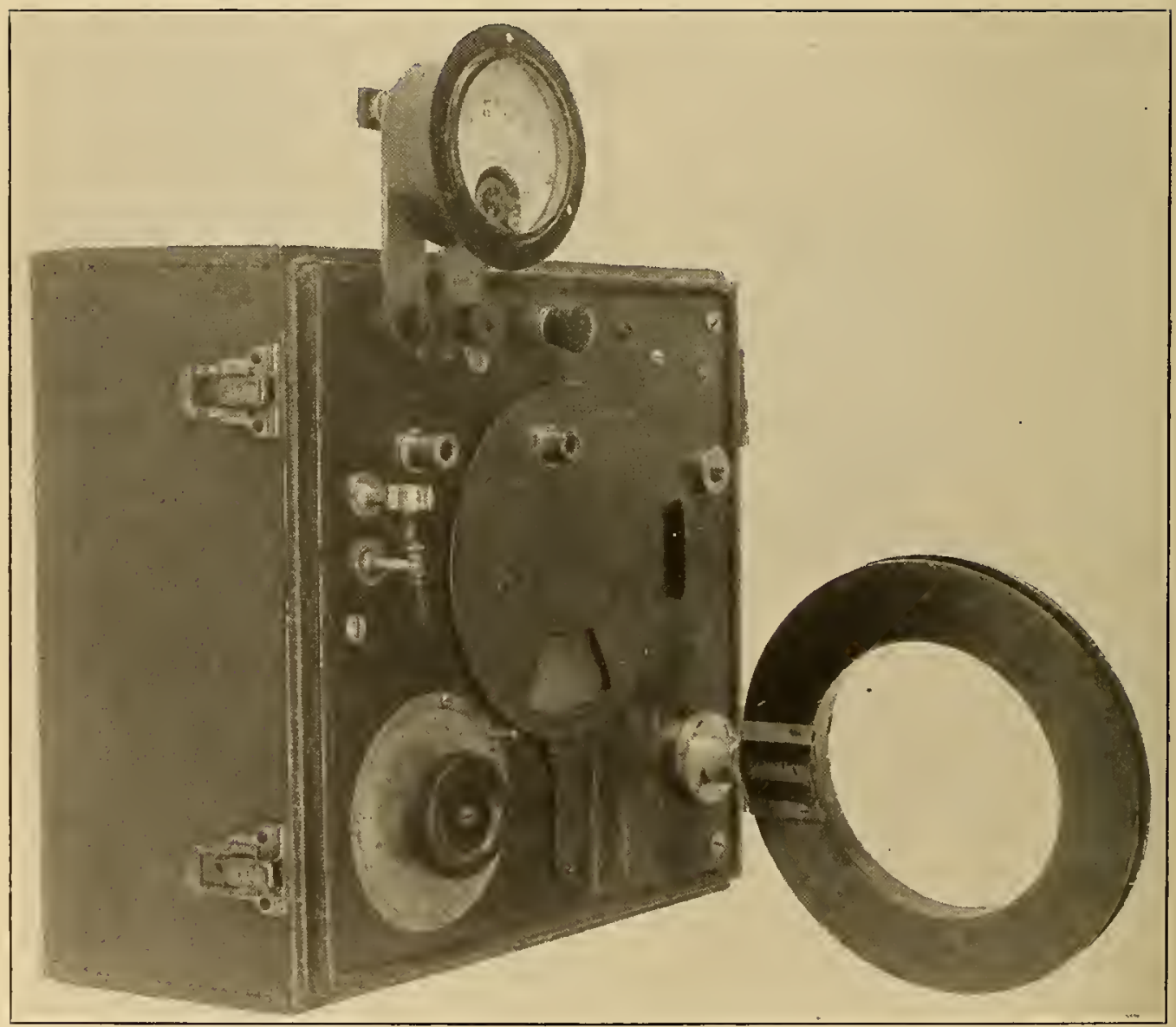

FIG. Th.-Exterior view of wave meter equipped with crystal milliammeter resonance indicator 
below. Two groups of frequencies were measured for each wavemeter coil-one at a high setting of the condenser and the other at a low condenser setting as indicated. Each group of frequencies is, of course, obtained by measurement from a source of radiofrequency power which remains constant in frequency. The first three frequencies in each group were measured when the crystal detector was adjusted to the point of maximum sensitivity; the other three frequencies were measured after readjusting the crystal detector to give a considerably lower deflection of the milliammeter at resonance.

These tabulated data show that precise measurements may be made over the frequency range of the wave meter and that a readjustment of the crystal detector does not affect the calibration of the wave meter.

\begin{tabular}{|c|c|c|c|c|}
\hline \multirow{2}{*}{ Coil } & \multicolumn{2}{|c|}{$\begin{array}{l}\text { Frequencies in kilocycles } \\
\text { as measured at approxi- } \\
\text { mately the highest cali- } \\
\text { brated setting of wave- } \\
\text { meter condenser }\end{array}$} & \multicolumn{2}{|c|}{$\begin{array}{l}\text { Frequencies in kilocycles a } \\
\text { measured at approxi } \\
\text { mately the lowest cali } \\
\text { brated sctting of wave- } \\
\text { meter condenser }\end{array}$} \\
\hline & $\begin{array}{l}\text { First adjust- } \\
\text { ment of } \\
\text { crystal de- } \\
\text { tector }\end{array}$ & $\begin{array}{l}\text { After read- } \\
\text { justment } \\
\text { of crystal } \\
\text { detector }\end{array}$ & $\begin{array}{c}\text { First adjust- } \\
\text { ment of } \\
\text { crystai de- } \\
\text { tector }\end{array}$ & $\begin{array}{l}\text { After read- } \\
\text { justment } \\
\text { of erystal } \\
\text { detector }\end{array}$ \\
\hline A. & $\begin{array}{l}960.5 \\
960.5 \\
960.5\end{array}$ & $\begin{array}{l}860.5 \\
960.1 \\
960.1\end{array}$ & $\begin{array}{l}1,415.2 \\
1,415.4 \\
1,415.2\end{array}$ & $\begin{array}{l}1,415.2 \\
1,415.4 \\
1,415.4\end{array}$ \\
\hline B. & $\begin{array}{l}679.2 \\
679.2 \\
679.1\end{array}$ & $\begin{array}{l}679.3 \\
679.3 \\
679.0\end{array}$ & $\begin{array}{l}985.7 \\
985.8 \\
985.9\end{array}$ & $\begin{array}{l}985.7 \\
985.7 \\
985.7\end{array}$ \\
\hline c. & $\begin{array}{l}550.0 \\
549.8 \\
549.9\end{array}$ & $\begin{array}{l}549.8 \\
550.0 \\
549.8\end{array}$ & $\begin{array}{l}810.6 \\
810.4 \\
810.5\end{array}$ & $\begin{array}{l}810.3 \\
810.6 \\
810.5\end{array}$ \\
\hline & $\begin{array}{l}380.1 \\
380.0 \\
380.2\end{array}$ & $\begin{array}{l}380.3 \\
380.2 \\
380.2\end{array}$ & $\begin{array}{l}555.5 \\
555.8 \\
555.6\end{array}$ & $\begin{array}{l}555.6 \\
555.8 \\
555.5\end{array}$ \\
\hline
\end{tabular}

\section{SUMMARY AND CONCLUSIONS}

A description has been given of a resonance indicating device for a portable wave meter which can be used to replace the thermogalvanometer. This device employs a crystal detector and milliammeter connected in such a manner that a combination of capacitive and inductive coupling is secured. It gives an approximately uniform resonance deflection throughout the frequency range of the wave meter, is more sensitive than the thermogalvanometer, and increases the precision of the wave meter. Tests have shown that this method of resonance indication is reliable.

The operation of a wave meter equipped with this resonance indicator is slightly less simple than that of a wave meter equipped with a thermogalvanometer, because an occasional adjustment of the crys- 
tal detector is required. If the detector is of good mechanical design and provided with a uniformly sensitive crystal, its adjustment is very easily made. The resonance indicator may be added to most portable wave meters and results in greater precision in their use.

Washington, August 13, 1924. 